\title{
Endoscopic Retrograde Cholangiopancreatography and Sedoanalgesia
}

\author{
Gulsah KARAOREN*, Nurten BAKAN*, Senay GOKSU TOMRUK ${ }^{\star}$ Kamil OZDIL ${ }^{* *}$, Seher ISIKER* \\ *Istanbul Education and Research Hospital, Anesthesiology and Reanimation \\ **Istanbul Education and Research Hospital, Gastroenterology
}

\section{Background:}

ERCP is a high quality but invasive procedure performed for diagnosis and treatment of biliary tract, pancreatic tract and periampullary region diseases.

Severe pain, hypertension, hypotension, bradycardia, desaturation, abdominal discomfort, dizziness are adverse events that can be seen during the procedure.

Risk factors for complications include known or unsuspected premorbid conditions, and problems related to anxiety, and insufficient analgesia. To facilitate the surgeon's work, to ensure patient safety and comfort, patients need to be sedated and followed closely with monitorisation.

For this purpose, remifentanil, propofol and/or dexmedetomidine infusions are preferred by anesthesiologists but It sometimes may be necessary to add benzodiazepine and narcotic support to the sedoanalgesia protocol for some cases with comorbidities.

In our study, it was aimed to compare different sedoanalgesia protocols of remifentanil, dexmedetomidine and propofol according to demographic characteristics, concomitant diseases, ASA score, operation time, additional narcotic and hypnotic dose.

\section{Material and Method:}

The study included patients aged $>18$ years in whom ERCP was indicated under sedo-analgesia.

The study was approved by Institutional Ethics Committee.

All patients gave written informed consent before participation. Analgesic agents were given 10 minutes before the ERCP procedure to provide sedation of 50-70 BIS level.

The study was designed with 4 groups.

Group 1 ( $\mathrm{n}=44$ ): 0.025-0.05 microgram $/ \mathrm{kg} / \mathrm{min}$ remifentanyl IV.

Group 2 ( $\mathrm{n=48):} 0.3$ microgram $/ \mathrm{kg} / \mathrm{h}$ dexmedetomidine IV.

Group 3( $n=125): 1-3 \mathrm{mg} / \mathrm{kg}$ propofol IV.

Group 4 ( $n=85): 0.5-1 \mathrm{mg} / \mathrm{kg}$ propofol IV bolus.

Demographic characteristics, BMI, Charlson comorbidity index, concomitant diseases, ASA score, operation time, additional narcotic and hypnotic dose and smoking were recorded.

\section{REFERENCES}

1) Turk J Anaesth Reanim. 2016; 44(1): 13-20

2) Gastrointest Endosc 2003;57: 633-8.
Results:

The study conducted on 302 patient (54\%,163 females and 46\%,139 males) with a mean age of $60.66 \pm 16.72$ years.

There was significant difference in the additional propofol use $(p=0.001 ; p<0.01)$ and midazolam use $(p=0.034 ; p<0.05)$ between groups

In binary comparison to identify source of difference, it was found that additional propofol use were significantly higher in group 2 than group $3(p=0.009 ; p<0.01)$ and additional dormicum use were significantly higher in group 2 than group 1 and group $3\left(p_{1}=0.013\right.$; $\left.\mathrm{p}_{2}=0.021 ; \mathrm{p}<0.05\right)$

No any other significant difference was detected ( $p>0.05)$.

Table 1: Demographic features and general data regarding to groups

\begin{tabular}{|c|c|c|c|c|c|}
\hline & & Min-Max & & Mean \pm SD & \\
\hline Age & & $22-90$ & & $60,66 \pm 16,72$ & \\
\hline BMI $\left(\mathrm{kg} / \mathrm{m}^{2}\right)$ & & $15,03-46,07$ & & $28,21 \pm 5,29$ & \\
\hline Operation Time ( $\mathrm{min})$ & & $10-180$ & & $41,9 \pm 24,07$ & \\
\hline Charlson Index & & $1-6$ & & $2,56 \pm 1,34$ & \\
\hline Propofol (mg) & & 10.460 & & $101,92 \pm 73,94$ & \\
\hline Fentanyl (mg) & & $25-80$ & & $54,9 \pm 13,11$ & \\
\hline \multirow[t]{2}{*}{ Dormicum (mg) } & & $1-4$ & & $1,71 \pm 0,63$ & \\
\hline & & $\mathrm{n}$ & & $\%$ & \\
\hline \multirow{2}{*}{ Gender } & Female & 163 & & 54 & \\
\hline & Male & 139 & & 46 & \\
\hline \multirow{4}{*}{ ASA } & 1 & 62 & & 20,5 & \\
\hline & 2 & 157 & & 52 & \\
\hline & 3 & 78 & & 25,8 & \\
\hline & 4 & 5 & & 1,7 & \\
\hline \multirow{3}{*}{ Additional Drugs } & Propofol & 279 & & 92,4 & \\
\hline & Fentanyl & 50 & & 16,6 & \\
\hline & Dormicum & 150 & & 49,7 & \\
\hline Diabetes Mellitus & & 56 & & 18,5 & \\
\hline Thyroid Disorder & & 30 & & 9,9 & \\
\hline $\mathrm{HT} / \mathrm{CAD} / \mathrm{CHF}$ & & 145 & & 48 & \\
\hline COPD/Astma & & 41 & & 13,6 & \\
\hline ARF/CRF & & 30 & & 9,9 & \\
\hline Neurologic Disorder & & 16 & & 5,3 & \\
\hline \multirow[t]{2}{*}{ Smoking } & & 92 & & 30,5 & \\
\hline & Group $1(\mathrm{n}=44)$ & Group $2(n=48)$ & Group $3(n=125)$ & Group 4 (n=85) & $p$ \\
\hline Age Mean SD & $63,68 \pm 15,13$ & $56,98 \pm 16,32$ & $62,27 \pm 17,09$ & $58,79 \pm 16,86$ & ${ }^{1} 0,112$ \\
\hline Female $_{n(\%)}$ & $24(\% 54,5)$ & $29(\% 60,4)$ & $69(\% 55,2)$ & $41(\% 48,2)$ & \multirow{2}{*}{${ }^{2} 0,570$} \\
\hline Male $_{n \beta G}$ & $20(\% 45,5)$ & $19(\% 39,6)$ & $56(\% 44,8)$ & $44(\% 51,8)$ & \\
\hline Propofol nipsi & $41(\% 93,2)$ & $48(\% 100)$ & $106(\% 84,8)$ & $84(\% 98,8)$ & ${ }^{2} 0,001^{* *}$ \\
\hline Fentanyl $_{n(\%)}$ & $10(\% 22,7)$ & $7(\% 14,6)$ & $16(\% 12,8)$ & $17(\% 20)$ & ${ }^{2} 0,340$ \\
\hline Dormicum $_{n(5 / f j}$ & $17(\% 38,6)$ & $32(\% 66,7)$ & $57(\% 45,6)$ & $44(\% 51,8)$ & ${ }^{2} 0,034^{*}$ \\
\hline
\end{tabular}

\section{Conclusion:}

ERCP is a process applied to high-risk patients with comorbidities. In our study, propofol infusion was found to be optimal during ERCP and dexmetodimidine was found insufficient. More large studies are needed to find the most suitable drug and suitable dose 